\title{
A novel locus for autosomal dominant, non-syndromic hearing impairment (DFNA18) maps to chromosome $3 q 22$ immediately adjacent to the DM2 locus
}

\author{
Dominikus Bönsch ${ }^{*, 1,2}$, Petra Scheer ${ }^{3}$, Cora Neumann ${ }^{1}$, Ruth Lang-Roth ${ }^{3}$, Eberhard Seifert ${ }^{3}$, \\ Peter Storch ${ }^{1,2}$, Cornelius Weiller ${ }^{2,4}$, Antoinette Lamprecht-Dinnesen ${ }^{3}$ and Thomas Deufel ${ }^{1}$
}

${ }^{1}$ Institut für Klinische Chemie und Laboratoriumsdiagnostik, Klinikum der Universität Jena, Jena, Germany;

${ }^{2}$ Klinik für Neurologie, Klinikum der Universität Jena, Jena, Germany; ${ }^{3}$ Poliklinik für Phoniatrie und

Pädaudiologie, Universität Münster, Münster, Germany

Investigating a large German pedigree with non-syndromic hearing impairment of early onset and autosomal dominant mode of inheritance, linkage to known DFNA loci was excluded and in a subsequent genomic scan the phenotype was mapped to a 10-cM interval on chromosome 3q22; a maximum two-point lod score of 3.77 was obtained for the marker D3S1292. The new locus, DFNA18, is excluded from neighbouring deafness loci, DFNB15 and USH3, and it overlaps with the recently described DM2/PROMM locus. As hearing loss has been described as one feature of the PROMM phenotype, the DFNA18 gene might also be responsible for hearing loss in DM2/PROMM. European Journal of Human Genetics (2001) 9, 165-170.

Keywords: hereditary hearing impairment; linkage analysis; chromosome 3q22; DFNA18; DM2 locus

\section{Introduction}

Hearing loss is the most frequent sensory defect in humans. About one in 1000 children is affected by prelingual deafness with severe deafness at birth or during infancy and a further one in 1000 children develop hearing impairment later in childhood; about $60 \%$ of these childhood cases are thought to be genetic. There exist syndromic and non-syndromic forms; about $75 \%$ of individuals present with hearing loss as the only clinical finding, the remaining $25 \%$ show additional features defining distinct clinical syndromes. ${ }^{1}$ The genetics of non-syndromic hearing loss are characterised by extensive genetic heterogeneity; to date, 31 autosomal dominant loci (DFNA), 28 recessive (DFNB) and eight $\mathrm{X}$-chromosomal

*Correspondence: D Bönsch, Klinik für Neurologie, Philosophenweg 3, 07743 Jena, Germany. Tel: +49 36419 35290; Fax: +49 36419 35025; E-mail: dominikus.boensch@med.uni-jena.de

${ }^{4}$ Current address: Klinik für Neurologie, Klinikum der Universität Hamburg, Hamburg, Germany

Received 10 January 2000; revised 7 November 2000; accepted 17 November 2000
(DFN) as well as mitochondrially transmitted forms have been reported. ${ }^{2}$ Despite the considerable difficulties in performing genetic mapping studies that arise from this heterogeneity, substantial progress was made in identifying genes involved in hearing loss. A number of genes have been cloned by now ${ }^{3-11}$ encoding for proteins of various functions such as myosin, transcription-factors or membrane proteins as connexins.

The DM2/PROMM locus was originally mapped to chromosome $3 \mathrm{q} 22^{12}$ neighbouring DFNA18, and a number of PROMM disease pedigrees have been mapped to the same locus and are considered allelic to DM2. ${ }^{13,14} \mathrm{DM} 2$ and PROMM are characterised by myotonia, proximal weakness and cataracts; in PROMM disease, hearing loss has been described as one clinical feature ${ }^{15}$ and is thus similar to myotonic dystrophy (DM1) located on chromosome 19, in which hearing loss has been reported as the only presenting symptom in some pedigrees, ${ }^{16}$ and where it has been suggested that CTG repeats within the DMPK gene may result in variable expression of different genes clustering within the region and thus produce a more complex phenotype. 


\section{Patients and methods}

\section{Clinical resources}

The probands investigated in this study belong to a large four-generation pedigree from the federal state of Westphalia in north-western Germany (Figure 1).

The family suffers from an autosomal dominant nonsyndromic progressive hearing loss. Onset of hearing loss is during the first decade of life and first involves the higher frequencies; in the next decades the middle and lower frequencies are also affected. The elderly affected members of the pedigree show a pantonal hearing threshold of about $80 \mathrm{~dB} \mathrm{nHL}$. The characteristic development of hearing loss is documented in Figure 2 which shows a representative hearing threshold of one member in each generation. None of the affected probands complained of tinnitus. The hearing impairment is classified as Type 2 according to Konigsmark and Gorlin ${ }^{17}$ or as Type I b according to Witkowski. ${ }^{18}$ There are also no signs of peripheral vestibular dysfunction or unsteadiness of gait in the dark. Decreasing visual fields and a progressive loss of visual acuity were ruled out by means of a questionnaire.

All affected probands underwent a neurological examination with a completely negative result; especially, no signs for an underlying muscle disease were observed. Four probands underwent a follow-up control 5 years after the initial examination. Otorhinolaryngological and neurological examinations still revealed no sign of associated abnormalities. However, the observation time was too short to demonstrate any relevant intraindividual deterioration of hearing.

The otorhinolaryngological examination included detailed microscopic otoscopy to rule out conductive hearing loss. The probands also underwent pure tone audiometry with air and bone conduction (Madsen OB 822 Clinical Audiometer or 622 D Audiometer respectively, Madsen, Denmark). To verify the cochlear genesis of hearing loss, otoacustic emissions (TEOAE) were measured (ILO 88 V4.20, Otodynamics Ltd., UK). Environmental factors or

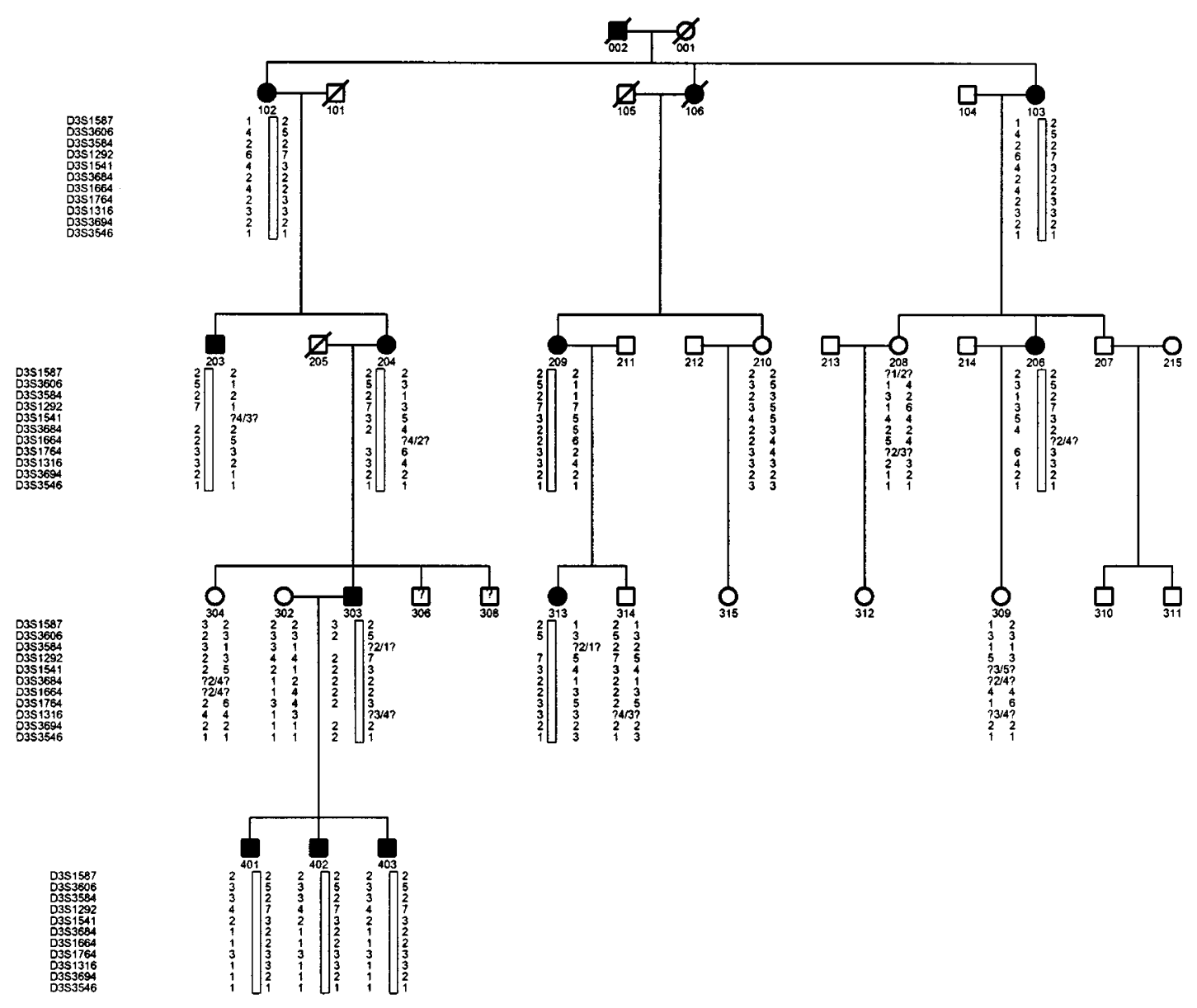

Figure 1 Pedigree of German family (MS-DFNA0094) with non-syndromic, progressive deafness. Filled symbols denote affected probands. The clinical status of proband 314 is uncertain and he has not been included in lod score calculations. For clinical details and markers see Fiegert et $a l^{19}$ and Patients and methods section. 
a

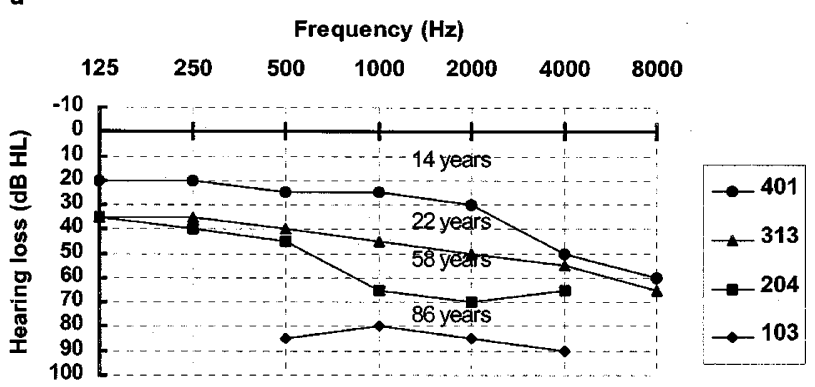

b

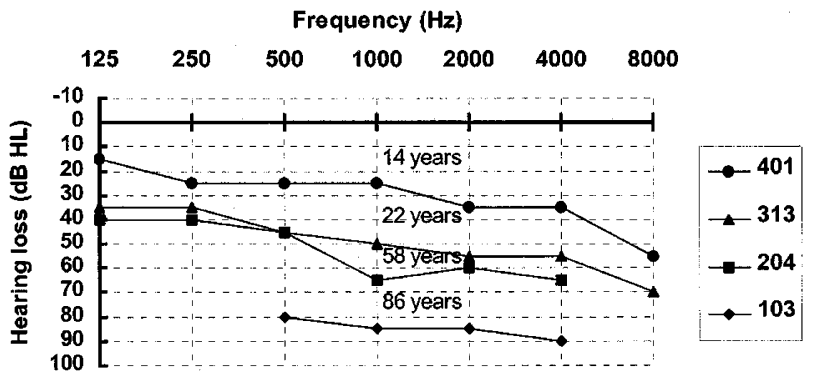

Figure 2 Typical audiograms from affected probands in pedigree MS-DFNA0094. Audiograms of probands from four generations (103, 204, 313 and 401 in Figure 1), right ear (a) and left ear (b).

other potential ototoxic influences which might have caused hearing loss were ruled out on the basis of a questionnaire. Probands with additional risk factors concerning hearing loss were excluded from further investigations. Probands were classified as affected only if the hearing loss was bilateral and symmetrical. Further clinical details have been described by Fiegert et al. ${ }^{19}$ All probands signed forms with all details of the study to give their informed consent.

Genotyping and linkage analysis

DNA was obtained from a total of 17 individuals, 11 affected and five unaffected sibs and one unaffected spouse. Genotyping for polymorphic microsatellite markers (see below) was performed by PCR following standard procedures. One primer of each pair was 5'-labeled with IRD 700 or 800 and products were analysed on a LICOR automated sequencing system (MWG Biotech). Alleles were scored and stored in table format according to a semi-automated protocol using RFLPscan and RFLP database software (Scanalytics). Two-point lod scores were calculated using the ILINK/MLINK programs of the LINKAGE software package (vers. 5.1). The order and distance between the marker loci were derived from the publicly accessible Marshfield Chromosome 3 Sex-Averaged linkage map.

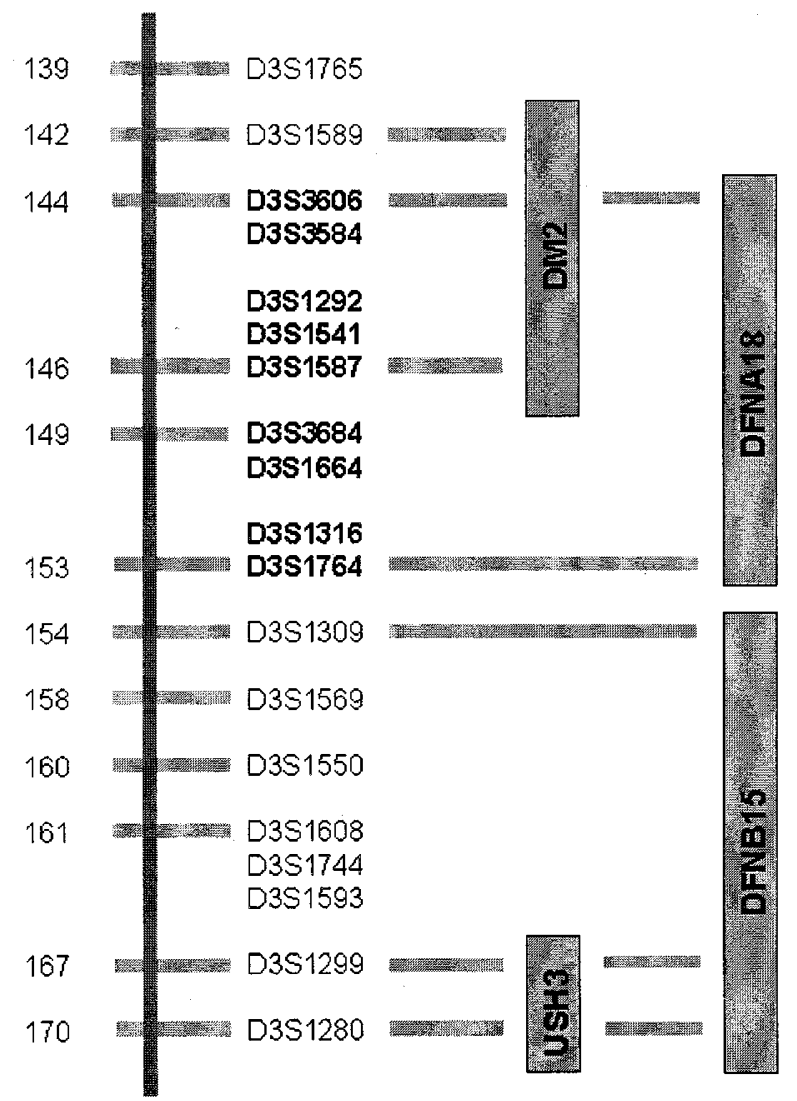

Figure 3 Integrated map of chromosome $3 q 22$ comprising the DFNA18 locus. The marker order corresponds to the Marshfield Chromosome 3 Sex-Averaged linkage map. Genetic distances are given in $\mathrm{CM}$. Markers shown in bold print are those with positive lod scores for DFNA18 (Table 1); the regions for DFNB15 and USH3 as well as DM2 are as reported. ${ }^{12,13,21,22,24}$

\section{Results}

Known loci for autosomal dominant hearing loss were excluded using reference markers described elsewhere; ${ }^{2}$ this was followed by a genome scan with a published set of 372 evenly spaced markers for autosomal chromosomes, ${ }^{20}$ resulting in a lod score of 3.77 at $\theta=0$ for index marker D3S1292, suggesting linkage, when about 300 markers had been examined. Additional markers from chromosome $3 \mathrm{q}$ as shown in Table 1 were typed in order to confirm linkage and for fine-mapping of the genetic interval. Positive lod scores were obtained for markers D3S1587, D3S3606, D3S3584, D3S1292, D3S1541, D3S3684, D3S1664, D3S1764, and D3S1316. One proband (marked 314 in Figure 1), originally diagnosed as clinically unaffected, shared most of the markers with his affected sister (313); as there remain doubts about his clinical state, however, he was not included in the linkage analysis at this time. Flanking markers with negative lod scores at $\theta=0$ are D3S1589 $(-\infty)$ and D3S1765 $(-2.08)$ on the 
Table 1

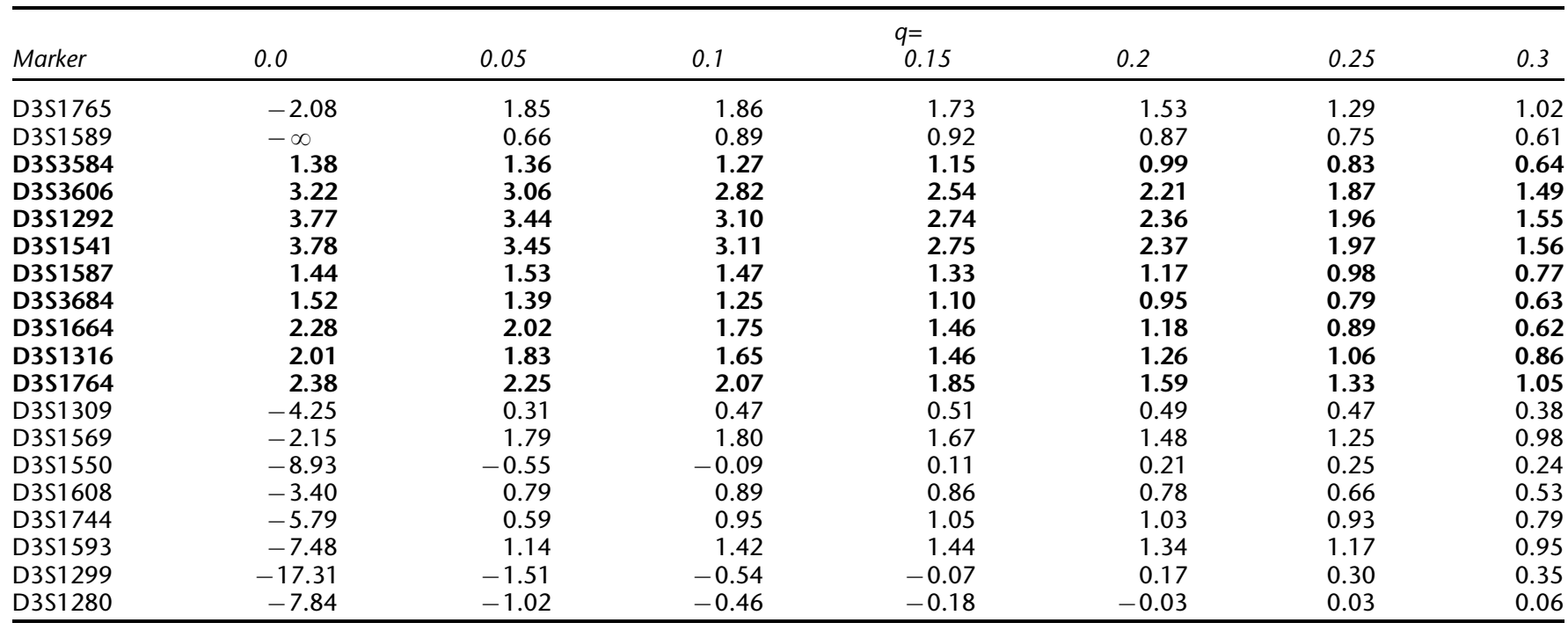

Pairwise lod scores were calculated for markers belonging to the DFNA18 region; the marker order corresponds to the Marshfield Chromosome 3 Sex-Averaged linkage map. Markers shown in bold print are those defining DFNA18.

centromeric side and D3S1569 (-2.15) and D3S1309 $(-4.25)$ on the telomeric side of DFNA18.

The loci for DFNB 15 and USH 3 are located in close proximity to the linked region (Figure 3 ) $;^{21-24}$ yet all published markers defining the DFNB15 and USH3 loci, the latter being comprised in DFNB15, have been excluded, and the absence of overlapping markers indicates that DFNA18 is indeed distinct from both these loci.

\section{Discussion}

The DFNA18 locus maps to chromosome 3q22 and is part of a cluster of deafness genes including DFNB15 and USH3

The novel DFNA18 locus for autosomal dominant, severe progressive hearing loss with onset in early childhood maps to chromosome 3q22 and thus in close proximity to, but distinct from the known deafness loci DFNB15 and USH3. The clinical findings of DFNA 18 are quite different from its surrounding loci. DFNB15 is an autosomal-recessive hearing disorder and the patients already have severe prelingual auditory impairment with profound hearing loss. The vestibular function is regular. ${ }^{23}$

In contrast, DFNA18 is characterised by a progressive hearing loss starting in the higher frequencies, and severe hearing loss is to be expected after the 5th decade. USH 3 again shows a progressive hearing loss with mild to moderate impairment in young patients and may become profound in adults but the patients suffer additionally from progressive vestibular areflexia while DFNA18 patients show no vestibular disorders. In addition to retinitis pigmentosa the patients with USH3 develop loss of visual acuity and nyctalopia. ${ }^{25,26}$

Our mapping of DFNA18 is thus a further example for the clustering of genes involved in hearing disorders as recently discussed by Van Hauwe ${ }^{27}$ for a similar cluster on chromosome $1 p$ in DFNA2 $2^{7,8}$ and as reported on chromosome $5 q$ for DFNA1 and DFNA15. ${ }^{3,5}$ The published marker order for this region is not fully consistent when comparing different maps available at the GDB homepage; detailed physical mapping is certain to change the marker order and allow for a more precise estimate of the genetic interval. To this end, a BAC and PAC contig is currently being assembled by screening pooled BAC (751 CEPH, Denis Le Paslier) and PAC (704 RCPI1, Pieter de Jong) gene banks obtained through the RZPD (DHGP, Berlin).

DFNA18 and DM2 map to adjoining genetic intervals and could contribute to a complex phenotype in DM2

The DM2 locus was originally mapped between D3S1589 and D3S1541, ${ }^{12}$ and a number of PROMM disease pedigrees have been mapped to the same locus and are considered allelic to DM2. ${ }^{13}$ For DFNA18, D3S1589 has been clearly excluded, yet the published DM2/PROMM and the DFNA18 regions overlap as indicated by the positive lod score we have obtained for D3S3606 and D3S1541. With positive lod scores for several other, more telomeric markers, DFNA18 reaches further to the q-telomeric end of chromosome 3 (Figure 3).

Comparing DM1 and DM2, an autosomal dominant locus for hearing impairment (DNFA4) has been found adjacent to the DM1 locus on chromosome $19 ;^{21}$ interestingly, hearing impairment has been described as part of the DM2/PROMM 
phenotype in some patients, ${ }^{15}$ again similar to findings in patients with classical DM1 where deafness has been described as the only presenting symptom in some patients. ${ }^{16}$

It has been discussed that the extension of the CTG repeat in the DMPK gene might produce a complex and possibly variable phenotype through its action on the expression of neighbouring genes. ${ }^{28,29}$ For ophthalmic symptoms, a feature present both in DM1 and DM2, involvement of several genes has already been shown ${ }^{30}$ and SIX5, a gene immediately downstream of DMPK seems to be involved in pathogenesis of cataract in DM1. Within the DFNA18 locus a gene responsible for adult and juvenile cataract (named BFSP2 or CP49 or phakinin) has recently been identified. ${ }^{31-33}$ Our findings of a deafness locus in this region might support a similar hypothesis for DM2/PROMM where the underlying mutation has not yet been identified.

Summarising, the hearing disorder in the pedigree investigated has been excluded from the known autosomal dominant loci DFNA1 to DFNA15 and maps to a new locus, DFNA18, on chromosome 3q22 close to but distinct from the known autosomal recessive locus DFNB15 as well as the USH 3 locus. DFNA18 is overlapping with the locus for DM2/ PROMM, a myotonic disorder where hearing loss has been reported as a prominent feature in some pedigrees. Several ESTs have already been mapped to this region, two of which (A005Y32 and WI-16414) represent cochlear transcripts ${ }^{10}$ and are thus currently being analysed as likely candidates for the DFNA18 gene.

\section{Acknowledgements}

This work has been supported by the DFG (Deutsche Forschungsgemeinschaft) (De 307/3-1 and 3-2).

\section{References}

1 Petit C: Genes responsible for human hereditary deafness: symphony of a thousand. Nature Genet 1998; 14: $385-391$.

2 Van Camp G, Smith RJH: Hereditary Hearing Loss Homepage (12/1999). World Wide Web URL: http://dnalab-www.uia.ac.be/ dnalab/hhh/

3 Lynch ED, Lee MK, Morrow JE, Welsch PL, Leon PE, King MC: Nonsyndromic deafness DFNA1 associated with mutation of a human homolog of the Drosophila gene diaphanous. Science 1997; 278: $1315-1318$.

4 Kelsell DP, Dunlop J, Stevens HP et al: Connexin 26 mutations in hereditary nonsyndromic sensorineural deafness. Nature 1997; 387: $80-83$.

5 Vahava O, Morell R, Lynch ED et al: Mutation in transcription factor POU4F3 associated with inherited progressive hearing loss in humans. Science 1998; 279: 1950-1954.

6 Verhoeven K, Van Laer L, Kirschhofer K et al: (1998). Mutations in the human $\alpha$-tectorin gene cause autosomal dominant nonsyndromic hearing impairment. Nature Genet 1998; 19: 60-62.

$7 \mathrm{Xia}$ JH, Liu CY, Tang BS et al: Mutations in the gene encoding gap junction protein beta-3 associated with autosomal dominant hearing impairment. Nature Genet 1998; 20: 370-373.
8 Kubisch C, Schroeder BC, Friedrich T et al: KCNQ4, a novel potassium channel expressed in sensory outer hair cells, is mutated in dominant deafness. Cell 1999; 96: 437-446.

9 Van Laer L, Huizing EH, Verstreken $M$ et al: Nonsyndromic hearing impairment is associated with a mutation in DFNA5. Nature Genet 1998; 20: $194-197$.

10 Robertson NG, Lu L, Heller S et al: Mutations in a novel cochlear gene cause DFNA9, a human nonsyndromic deafness with vestibular dysfunction. Nature Genet 1998; 20: 299-303.

11 Liu XZ, Walsh J, Tamagawa Y et al: Autosomal dominant nonsyndromic deafness caused by a mutation in the myosin VIIA gene. Nature Genet 1997; 17: $268-269$.

12 Ranum LP, Rasmussen PF, Benzow KA, Koob MD, Day JW. Genetic mapping of a second myotonic dystrophy locus. Nature Genet 1998; 19: 196-198.

13 Ricker K, Grimm T, Koch MC et al: Linkage of proximal myotonic myopathy to chromosome 3q. Neurology 1999; 52: $170-171$.

14 Ricker K. Myotonic dystrophy and proximal myotonic myopathy. J Neurol 1999; 246: 334-338.

15 Udd B, Krahe R, Wallgren-Pettersson C, Falck B, Kalimo H: Proximal myotonic dystrophy - a family with autosomal dominant muscular dystrophy, cataracts, hearing loss and hypogonadism: heterogeneity of proximal myotonic syndromes? Neuromusc Disord 1997; 7: 217-228.

16 Wright RB, Glantz RH, Butcher J. Hearing loss in myotonic dystrophy. Ann Neurol 1988; 23: 202-203.

17 Konigsmark WB, Gorlin RJ: Genetic and metabolic deafness. Saunders: Philadelphia, London, Toronto 1976: pp 7-35.

18 Witkowski R, Prokop O, Ullrich E: Lexikon der Syndrome und Fehlbildungen. Springer Verlag: Berlin, Heidelberg 1999: p 1079.

19 Fiegert P, Lamprecht-Dinnesen A, Preisler-Adams S, Deufel T: Molekulare Analysen der Heterogenität hereditärer monosymptomatischer Schallempfindungs-schwerhörigkeiten. LaryngoRhino-Otolaryngology 1996; 75: $141-147$.

20 Dubovsky J, Sheffield VC, Duyk GM, Weber JL: Sets of short tandem repeat polymorphisms for efficient linkage screening of the human genome. Human Mol Genet 1995; 4: 449-452.

21 Chen AH, Ni L, Fukushima $\mathrm{K}$ et al: Linkage of a gene for dominant non-syndromic deafness to chromosome 19. Human Mol Genet 1995; 4: 1073-1076.

22 Chen A, Wayne S, Bell A et al: New gene for autosomal recessive non-syndromic hearing loss maps to either chromosome $3 \mathrm{q}$ or 19p. Am J Med Genet 1997; 71: 467-471.

23 Chen AH, Fukushima K, McGuirt WT, Smith RJ: DFNB 15: Autosomal recessive non-syndromic hearing loss gene-chromosome 3q, 19p digenic recessive inheritance? Advances Otorhinolaryngol 2000; 56: 171-175.

24 Joensuu T, Blanco G, Pakarinen L et al: Refined mapping of the Usher syndrome type III locus on chromosome 3, exclusion of candidate genes, and identification of the putative mouse homologous region. Genomics 1996; 38: 255-263.

25 Kimberling WJ, Orten D, Pieke-Dahl S: Genetic heterogeneity of usher syndrome; in Kitamura K, Steel KP (eds): Genetics in Otorhinolaryngology. Adv Otorhinolaryngol Basel 2000, vol 56: pp $11-18$.

26 Keats B, Corey DP: The Usher Syndromes. Am J Med Genet 1999; 89: $158-166$

27 Van Hauwe P, Coucke PJ, Declau F: Deafness linked to DFNA2: one locus but how many genes? Nature Genet 1999; 21: 263.

28 Groenen P, Wieringa B: Expanding complexity in myotonic dystrophy. Bioessays 1998; 20: 901-912.

29 Korade-Mirnics Z, Babitzke P, Hoffman E: Myotonic dystrophy: molecular windows on a complex etiology. Nucleic Acids Res 1998; 26: $1363-1368$. 
30 Winchester CL, Ferrier RK, Sermoni A, Clark BJ, Johnson KJ: Characterization of the expression of DMPK and SIX5 in the human eye and implications for pathogenesis in myotonic dystrophy. Human Mol Genet 1999; 8: 481-492.

31 Kramer PL, LaMorticella D, Schilling K, Biilingslea AM, Weleber RG, Litt M: A new locus for autosomal dominant congenital cataracts maps to chromosome 3. Invest Ophthalmol Visual Sci 2000; 41: 36-39.

32 Jakobs PM, Hess JF, FitzGerald PG, Kramer PL, Weleber RG, Litt M: Autosomal-dominant congenital cataract associated with a deletion mutation in the human beaded filament protein gene BFSP2. Am J Human Genet 2000; 66: 1432-1436.

33 Carter JM, McLean WHI, West S, Quinlan RA: Mapping of the human CP49 gene and identification of an intragenic polymorphic marker to allow genetic linkage analysis in autosomal dominant congenital cataract. Biochem Biophys Res Commun 2000; 270: 432-436. 